\title{
Alfabetización financiera en jóvenes en Ecuador: modelo de medición y sus factores determinantes
}

\author{
Carola L. Mena-Campoverde \\ Facultad de Economía y Administración de Empresas, Universidad Católica de Santiago de Guayaquil, Km. 1.5 vía \\ Carlos Julio Arosemena, Guayaquil, Ecuador. \\ (correo: carola.mena@cu.ucsg.edu.ec; carolamenacampoverde@gmail.com)
}

Recibido Jun. 9, 2021; Aceptado Jul. 29, 2021; Versión final Sept. 25, 2021, Publicado Feb. 2022

\begin{abstract}
Resumen
El propósito de esta investigación fue determinar un modelo de medición de alfabetización financiera y validación de constructo y obtener los niveles de alfabetización financiera y los factores sociodemográficos que marcan la diferencia de niveles. Se aplicó un modelo de ecuaciones estructurales con una muestra de 420 jóvenes en Ecuador. Los resultados mostraron que la alfabetización financiera es un constructo multidimensional. Se determinó un modelo adecuado en el que la alfabetización financiera se explica por los comportamientos financieros, actitudes financieras y conocimientos financieros. Los factores socioeconómicos y demográficos que marcan diferencias de niveles de alfabetización financiera fueron el nivel de educación, nivel de ingresos, estado civil, tipo de actividad y los comportamientos financieros de los padres y la educación financiera que ellos les proveen a sus hijos. Se concluye que la alfabetización financiera es un constructo multidimensional que se explica por conocimientos financieros, actitudes financieras y comportamientos financieros.
\end{abstract}

Palabras clave: alfabetización financiera; modelo; ecuaciones estructurales; factores sociodemográficos

\section{Financial literacy in youth from Ecuador: measurement model and its determining factors}

\begin{abstract}
The purpose of this research study was to develop a measurement model for financial literacy and validation of construct to assess financial literacy levels and the socio-demographic factors that determine them. A structural equation model was applied using a sample of 420 young Ecuadorians. The results showed that financial literacy was a multidimensional construct. An adequate model was developed in which financial literacy was best explained by financial behaviors, financial attitudes, and financial knowledge. The socioeconomic and demographic factors that determined the differences between financial literacy levels were: educational level, income level, marital status, activity type, parent's financial behavior, and parent's financial education to their children. It is concluded that financial literacy is a multidimensional construct explained by financial knowledge, financial attitudes, and financial behaviors.
\end{abstract}




\section{INTRODUCCIÓN}

Mejorar la alfabetización financiera de los consumidores es de interés para los gobiernos de todo el mundo. El comprender cómo los individuos toman sus decisiones financieras ha sido motivo de preocupación de la comunidad científica. Para la OCDE la alfabetización financiera "es el proceso por el cual los consumidores/inversionistas financieros mejoran su comprension de los productos financieros, los conceptos y los riesgos, y, a traves de informacion, instrucción y/o el asesoramiento objetivo, desarrollan las habilidades y confianza para ser mas conscientes de los riesgos y oportunidades financieras, tomar decisiones informadas, saber a dónde ir para obtener ayuda y ejercer cualquier accion eficaz para mejorar su bienestar economico" (OECD, 2011).

Estudios recientes sobre la alfabetización financiera nos dan cuenta de su relevancia para mejorar la calidad de vida de los habitantes (Kamakia et al., 2017). Acorde a la evidencia empírica, quienes tienen mejores niveles de alfabetización financiera, son más propensos al ahorro (Widjaja et al., 2020) e inversiones (Raut, 2020) y a manejar adecuadamente sus niveles de endeudamiento (French y McKillop, 2016). Dada la importancia de la alfabetización financiera para el crecimiento y estabilidad económica a nivel global, la OECD recomienda realizar mediciones periódicas de niveles de alfabetización financiera con encuestas adaptadas al entorno financiero de cada país (OECD, 2021), sin embargo, esta tarea no ha sido nada fácil en especial en Latinoamérica, puesto que no existe un acuerdo en la comunidad académica o científica sobre la definición de constructo de la alfabetización financiera ni de los instrumentos de medición y la mayor parte de estudios relacionados se han realizado en países desarrollados donde las realidades son muy diferentes a países emergentes.

Sobre ésta problemática, en el 2010, Huston analizó 71 estudios de alfabetización financiera, la mayoría fueron realizados en Estados Unidos, y sólo el 13\% de estas investigaciones definieron constructos; en las encuestas realizadas, han existido desde 3 ítems hasta 68, en promedio 13; el 68\% de las encuestas se dirigieron a target específico, los tamaños de muestras fueron desde 42 hasta 12,140 (Huston, 2010), también identificó ocho definiciones distintas de alfabetización financiera, el $47 \%$ de las investigaciones analizadas habían usado como sinónimo los términos Alfabetización Financiera y Educación Financiera, el 90\%, no dio indicador de lo que corresponde denominar a una persona como alfabetización financiera, revelando que "hay tres importantes barreras para desarrollar una medida estandarizada de alfabetización financiera: la falta de conceptualización y definición de constructo de alfabetización financiera, contenido de los instrumentos de medida, e interpretación de los instrumentos. La primera barrera es la más importante" (Huston, 2010, p. 305).

La presente investigación se orienta justamente a cerrar las brechas mencionadas, más aún que hay pocos estudios sobre el tema en Latinoamérica adaptados a las casuísticas propias su entorno financiero como lo recomienda realizar la OCDE. En Latinoamérica, un trabajo pionero en la definición de constructo y desarrollo de medida estandarizada de alfabetización financiera fue realizado en Brasil en una muestra de 991 estudiantes universitarios por Potrich et al. (2015), en el que se explicó a la alfabetización financiera por medio de las dimensiones: comportamiento financiero, actitudes financieras y conocimientos financieros, para ello utilizaron un cuestionario que se basó en la revisión de literatura, utilizaron un modelo factorial confirmatorio para verificar las preguntas que se ajustaban a cada dimensión, y finalmente aplicaron un modelo de ecuaciones estructurales que mostró tener buen ajuste, pero para ello tuvieron que eliminar algunas preguntas que no aportaban significativamente a las dimensiones ni al modelo, quedando una escala de medición fiable así como una conceptualización multidimensional de alfabetización financiera que está contextualizada a los jóvenes universitario en Brasil.

En el 2015 la OCDE realizó una encuesta sobre capacidades financieras para sus países miembros y otros (Lusardi, 2015) en el que China obtuvo 603 puntos; USA, 492; España, 484; y el promedio de los países de la muestra fue de 500 puntos; el único país de Latinoamérica en el estudio fue Colombia, que fue el peor puntuado de todo el estudio con 379 puntos, sin embargo, de cara a la encuesta de capacidades financieras realizada por la CAF en el 2014 para países andinos (Mejía et al., 2015), Colombia fue el mejor puntuado, pero como vemos, cuando compite con países miembros de la OCDE, es el último en la lista; acorde al informe de la CAF, pocos encuestados conocen de interés simple, y muchos prefieren guardar dinero en sus casas, en el caso de Ecuador, el $41 \%$ de la población no ahorra y que de hacerlo no lo realizan en instituciones financieras, sino bajo modalidades rústicas ("debajo del colchón”).

Diez Martínez (2016) hizo un diagnóstico de los niveles de conocimiento financieros de adolescentes mexicanos, encontrando que los niveles fueron bajos; similares resultados obtuvieron Moreno et al. (2017) en jóvenes universitarios de carreras administrativas del mismo país. En Chile, Denegri Coria et al. (2016) encontraron que, a pesar de habérseles impartido un programa de alfabetización económica a un grupo de estudiantes universitarios, los niveles de alfabetización económica continuaron bajos, sin embargo, en este estudio se debe recalcar que la escala de medición de alfabetización económica presentó baja confiabilidad 
estadística, por lo que recomiendan realizar más estudios sobre ello. En Bolivia, Garay Anaya (2016) realizó una medición de alfabetización financiera basándose en las escalas de conocimientos financieros y actitudes financieras de investigaciones previas, si bien ambas escalas mostraron buenos niveles de confiabilidad, no se incorporó la dimensión "comportamiento financiero" ni se realizó una validez de constructo de alfabetización financiera, y las preguntas tenían escala de Likert de 10 puntos, lo que no es común en los estudios de alfabetización financiera sino hasta 5 , y al igual que en las investigaciones previamente mencionadas, los niveles de conocimiento financiero fueron bajos.

En el 2021, Mungaray et al., realizaron un estudio para establecer la implicancia de la alfabetización financiera en el ingreso, tomando una muestra de la población mexicana de personas en edades entre 18 a 70 años. En el estudio le llaman a la alfabetización financiera "educación financiera", siendo dos conceptos diferentes; los investigadores construyeron una escala de medición de educación financiera basándose en previas investigaciones en países desarrollados, la escala analizó comportamientos, actitudes y conocimientos financieros, encontrando una asociación sólo de 1.75\% entre el ingreso y el índice de educación financiera, pero la construcción del índice no contó con validación de fiabilidad de las escalas, los cuestionarios para comportamiento financiero y actitud financiera no se realizaron con el uso de escala de Likert sino dando un puntaje de 1 a respuesta correcta y 0 para incorrecta, no dejando al encuestado términos medio, por ejemplo "ahorro activo" una persona puede que no ahorre todos los meses pero si pasando un mes, por otro lado el cuestionario en términos de actitudes financieras sólo tenía dos ítems a evaluar, probablemente por ello la relación mostrada del índice de alfabetización financiera con el ingreso, es tan bajo.

Además de estudios en países de Latinoamérica, se han venido realizando investigaciones al respecto en otros países como en Ghana, donde Despard y Chowa (2014) testearon un modelo de medición de capacidades financieras en una muestra de adolescentes de 12 a 18 años, donde utilizaron un modelo factorial exploratorio y un confirmatorio, quedando como resultado un instrumento de 12 ítems que inicialmente era de 18, con tres dimensiones: Manejo del dinero, Conciencia de servicios financieros y Acciones de servicios financieros. Recientemente en Indonesia, Dewi et al. (2020), realizaron un modelo de medición de capacidades financieras en la comunidad académica, usando un modelo de ecuaciones estructurales, que como resultado se obtuvo que las capacidades financieras son explicadas por el conocimiento financiero y las habilidades financieras, y éstas a su vez, por la conciencia financiera y la experiencia financiera, además el modelo indicó que las capacidades financieras explican a las metas financieras, al comportamiento financiero y a las decisiones financieras, el cuestionario aplicado contó con 34 preguntas y se aplicó a una muestra de 889 docentes.

Acorde a los trabajos aquí revisados, los niveles de conocimientos financieros en Latinoamérica son bajos, se utiliza intercambiablemente el término "alfabetización financiera" con "capacidades financieras", "educación financiera", entre otros, también evidencian que falta una definición clara de alfabetización financiera y una escala de medida lo más estandarizada posible acorde al contexto latinoamericano, que permita realizar mediciones periódicas de alfabetización financiera, así como identificar cuáles son los factores sociodemográficos que puedan tener también incidencia en la alfabetización financiera, lo cual hace justificable la presente investigación.

\section{METODOLOGIA}

El enfoque utilizado en esta investigación fue cuantitativo, tipo de estudio exploratorio y causal (modelo de ecuaciones estructurales), tipo de muestreo aleatorio simple y estratificado por jóvenes de 18 a 28 años, de las zonas urbanas de la ciudad de Guayaquil. El cuestionario utilizado se adaptó al contexto de inclusión financiera de Ecuador donde no hay un mercado financiero y de valores amplio y diversificado y se validó el cuestionario con expertos del Instituto de Investigaciones Económicas de la Universidad Católica de Santiago de Guayaquil, dejando sólo las preguntas que más se acercan al contexto financiero de Ecuador, tomando como base el cuestionario que usaron Potrich et al. (2015) en un estudio similar en Brasil. Se utilizó un cuestionario de 5 puntos en escala de Likert. En cuanto a los temas de conocimiento financiero se incluyeron las preguntas desarrolladas por Lusardi y Mitchell (2008, 2011a).

Las dimensiones evaluadas para definir el constructo "Alfabetización financiera" fueron: Conocimientos financieros, Actitudes financieras, Comportamiento financiero. Para evaluar conocimientos financieros, se utilizaron las tres preguntas bien conocidas de Lusardi (2015) sobre interés simple, interés compuesto e inflación aplicado a valor de dinero en el tiempo (conocimiento financiero básico) y también se añadieron dos preguntas sobre conocimientos financieros avanzados (rentabilidad-riesgo de instrumentos financieros y diversificación del riesgo). Las preguntas utilizadas para conocimientos financieros básicos fueron: 1. Imagine que usted tiene $\$ 100$ en su cuenta de ahorros ganando interés de $10 \%$ anual, luego de 5 años, ¿cuánto dinero tendrá en su cuenta? a) Menos de 150, b) Más de 150, c) Igual. Respuesta: Más de 150. 2. Imagine que la tasa de interés aplicada a sus ahorros es del $6 \%$ y que la inflación es del $10 \%$ anual, luego de un año, ¿cuánto 
podrá comprar con el dinero de esta cuenta? a) Más que hoy, b) Menos que hoy, c) Igual que hoy. Respuesta: Menos que hoy. 3. Imagine que José hereda $\$ 10,000$ hoy, y Pedro hereda $\$ 10,000$ en tres años, ¿quién tendrá más? a) José, b) Pedro, c) Iguales. Respuesta: José. Las preguntas para conocimientos financieros avanzados fueron: 1. En el largo plazo, ¿cuál de los siguientes instrumentos financieros da mayor retorno? a) Acciones, b) Bonos, c) Cuentas de ahorros. Respuesta: Acciones. 2. Cuando se diversifica, el riesgo de perder dinero, a) Aumenta, b) Disminuye, c) Ninguna de las anteriores. Respuesta: Disminuye.

En lo referente a actitudes financieras y comportamiento financiero, se evaluaron actitudes hacia el ahorro, inversiones, manejo de presupuesto, manejo de deudas, conciencia de repercusiones a futuro del manejo financiero de hoy, buen uso de tarjeta de crédito, fondo de emergencia; y sobre información sociodemográfica: género, nivel de educación, nivel de ingresos, tipo de trabajo (como empresario o empleado), estado civil, número de hijos, edad, actividad que realiza, comportamiento financiero de los padres, enseñanza de finanzas de padres.

Se aplicaron las encuestas a 420 jóvenes bajo consentimiento informado, en un proceso cara a cara, previo a ello, se realizó un test de validez de las escalas del cuestionario con una muestra de 30 encuestas, por medio del Alpha de Cronbach, se obtuvo como resultado un indicador de fiabilidad de 0.754 y 0.854 para Actitud Financiera y Comportamiento financiero respectivamente, y una varianza de 11.49 y 56.59 para ambos constructos, el constructo es considerado confiable si la el puntaje de confiabilidad y la varianza extraída es igual o mayor que 0.7 y 0.5 respectivamente (Hair et al., 2009). Acorde a los descriptivos de la muestra, el $61 \%$ está entre las edades de 18 a 24 años y el $39 \%$ de 25 a 28 años; $52.6 \%$ de la muestra son mujeres; el $79.8 \%$ es soltero; el $80 \%$ no tiene hijos; el $45 \%$ sólo se dedica a estudiar la Universidad, $26.2 \%$ sólo trabaja, y el $24.5 \%$ ambos a la vez, el $79 \%$ ha culminado el bachillerato y el $20.5 \%$ ya cuenta con un grado profesional.

Una vez recogida la muestra, se realizó un análisis factorial confirmatorio para las dimensiones "Comportamiento Financiero" y "Actitudes financieras", luego se realizó una validez de constructo usando un modelo de ecuaciones estructurales, de acuerdo a Byrne (2010), esta es una técnica estadística que estima y prueba relaciones causales entre variables basadas en data estadística e hipótesis causales cuantitativas, es considerado un análisis multivariado porque permite la consideración de relación entre constructos dependientes e independientes de manera simultánea.

Los valores estadísticos a utilizar para validar un modelo de ecuaciones estructurales acorde a Byrne (2010) y también según Escobedo y Hernández (2016) son: bondad de ajuste absoluto que determina el grado en que el modelo general predice la matriz de correlaciones y para SEM, el estadístico-radio de verosimilitud Chicuadrado es la única medida estadística. El modelo tiene un ajuste aceptable si los valores de Chi-cuadrado/gl son de 2 a 3 y con límites de hasta 5 . El Índice de bondad de ajuste (GFI) evalúa si el modelo debe ser ajustado. Entre más se acerque a cero indica un mal ajuste. El error de aproximación cuadrático medio (RMSEA), representa el ajuste anticipado con el valor total de la población y ya no con el de la muestra. Si RMSEA es menor o igual a 0.05 indica un error de aproximación del modelo con la realidad (Escobedo y Hernández, 2016).

Después de obtener el modelo, se creó un índice de alfabetización financiera con las preguntas que quedaron validadas por el modelo y se categorizó a los encuestados en diferentes niveles de alfabetización financiera, para finalmente realizar un análisis discriminante de niveles de alfabetización financiera por factores sociodemográficos. Para calcular el indicador, en las preguntas de escala de Likert de 5 puntos de las dimensiones "Actitud Financiera" y "Comportamiento Financiero", se obtuvo un promedio de los puntajes de las preguntas que componían cada escala, y se catalogó como nivel "Bajo" a aquellos promedios que iban de 1 a 2; "Medio", aquellos de 3 a 4; y, "Alto" a los de valor 5 . En el caso de conocimientos financieros, cada una de las cinco preguntas bien contestada valía un punto. Finalmente, el indicador de Alfabetización financiera fue un promedio de las tres dimensiones analizadas, y se hizo la misma categorización que con cada dimensión.

\section{RESULTADOS Y DISCUSION}

Sobre los resultados descriptivos, en cuanto a actitudes financieras, un $71.7 \%$ de los encuestados considera que es importante controlar los gastos cada mes; el $66 \%$ considera que es importante fijarse metas financieras para el futuro; el 53\% considera estar totalmente de acuerdo en que se debe seguir un presupuesto. Sobre comportamiento financiero, sólo el 35\% ahorra mensualmente y lleva un registro de gastos personales, el $53 \%$ paga sus deudas a tiempo, y sólo el $29.8 \%$ se encuentra totalmente satisfecho con la manera en que maneja sus finanzas, comparando estos datos con la encuesta de la OCDE en el 2018 (OECD, 2021) en promedio de todos países encuestados, el $70 \%$ de los adultos son ahorradores activos, en Colombia sólo el $40 \%$ ahorra, en Indonesia lo hace el $99.7 \%$. Como vemos, el comportamiento financiero de los jóvenes 
entrevistados está por debajo de los de los países vecinos Colombia y Perú, y más aún del promedio de países del estudio de la OCDE.

Relacionado a conocimientos financieros, los resultados (Tabla 1) no fueron distintos a los de muchos países donde son bajos, con excepción de interés simple (la primera pregunta), en el resto de las preguntas la mayor proporción fueron respuestas incorrectas. En la encuesta de alfabetización financiera de la OCDE el 84\% contestaron acertadamente la pregunta sobre interés simple mientras que en este estudio sólo $59 \%$; sobre la inflación aplicada a valor de dinero en el tiempo el 38\% contestó acertadamente, y en la de la OCDE fueron acertadas en un $49.7 \%$ de Colombia, y $55.2 \%$ de Perú; sobre riesgo-diversificación, sólo el $32 \%$ de los jóvenes acertaron mientras que en la de la OCDE el $58.9 \%$.

Tabla 1: Preguntas acertadas y fallidas sobre conocimientos financieros:

\begin{tabular}{|l|c|c|c|c|c|}
\cline { 2 - 6 } \multicolumn{1}{c|}{} & \multicolumn{4}{c|}{ Conocimiento Financiero (Porcentaje) } \\
\cline { 2 - 6 } & \multicolumn{3}{c|}{ Conoc. Financ. Básico } & \multicolumn{2}{c|}{ Conoc. Financ. Avanz. } \\
\cline { 2 - 6 } & Interés simple & Interés comp. & Inflación & Instrum. Financ & Diversif. \\
\hline Correctas & 58.8 & 43.6 & 38.3 & 42.1 & 32.4 \\
\hline Incorrectas & 41.2 & 56.4 & 61.7 & 57.9 & 67.6 \\
\hline Total & 100 & 100 & 100 & 100 & 100 \\
\hline
\end{tabular}

Sobre el comportamiento financiero de los padres o familiar más cercano, sólo el $37 \%$ de ellos siempre ahorran cada mes y gasta acorde a un presupuesto, apenas el $26 \%$ realizan inversiones en el largo plazo, y sólo el $47 \%$ les enseñan a los jóvenes sobre la importancia del ahorro, lo cual preocupa, ya que de acuerdo a Hanson y Olson (2018) las conversaciones sobre finanzas que se tienen dentro del hogar de padres a hijos, ayuda a elevar el nivel de conocimientos financieros y a impulsar actitudes y conductas positivas hacia el ahorro e inversión; acorde a Hernández et al. (2017), los padres influyen mucho en la educación de sus hijos, en especial las madres, destacando que los factores motivacionales ayudan a tener mejor resultados. Por tanto, es importante que los programas de alfabetización financiera contemplen la participación activa de los padres para mejorar los niveles de alfabetización financiera de sus hijos.

\section{Modelo de medición de alfabetización financiera}

Para confirmar los factores que pertenecen a las dimensiones evaluadas, se realizó un análisis factorial confirmatorio evaluando "Comportamiento Financiero" y "Actitudes financieras"; en un primer paso no se obtuvo un modelo fiable para ambos constructos, por tanto, se eliminaron aquellas preguntas que distorsionaban el modelo de cada dimensión, dejando un modelo final fiable para cada dimensión. (Tabla 2).

Tabla 2: Resultados del Análisis Factorial Confirmatorio para Comportamiento Financiero y Actitudes Financieras

\begin{tabular}{|l|c|c|c|c|}
\hline \multirow{2}{*}{\multicolumn{1}{|c|}{ Indicadores de Bondad de Ajuste }} & \multicolumn{2}{c|}{ Comportamiento Financiero } & \multicolumn{2}{c|}{ Actitud Financiera } \\
\cline { 2 - 5 } & Propuesto & Final & Propuesto & Final \\
\hline GFI- Goodness of Fit & 0.971 & 0.98 & 0.977 & 0.989 \\
\hline CFI- Comparative Fit Index & 0.892 & 0.991 & 0.975 & 0.991 \\
\hline NFI-Normed Fit Index & 0.876 & 0.979 & 0.964 & 0.984 \\
\hline TLI-Tuker Lewis Index & 0.856 & 0.986 & 0.959 & 0.982 \\
\hline RMSR- Root Mean Square Residual & 0.11 & 0.029 & 0.035 & 0.016 \\
\hline RMSEA-RMS Error of Approximation & 0.115 & 0.039 & 0.69 & 0.054 \\
\hline Conbach's Alpha & 0.856 & 0.79 & 0.79 & 0.807 \\
\hline CMIN/DF & 6.53 & 1.635 & 3.01 & 2.212 \\
\hline
\end{tabular}

En la Tabla 3 se encuentran las preguntas que ajustaron en cada escala, además de éstas preguntas el cuestionario final tendrá las de Conocimiento Financiero, y con todas ellas se testeó el modelo de ecuaciones estructurales.para determinar la validez del constructo "Alfabetización Financiera" en AMOS 23. Como resultado se determinó un modelo fiable de alfabetización financiera para jóvenes en la ciudad de Guayaquil, Ecuador, se explica por conocimientos financieros, comportamientos financieros (básicos y avanzados) y actitudes financieras. (Tabla 4). Estos resultados son consistentes con estudios de (Potrich et al., 2015; Rai et al., 2019; Potrich et al., 2016) en los cuales se han desarrollado modelos de alfabetización financiera evaluando las dimensiones de conocimiento financiero, actitudes financieras y comportamiento financiero como explicativas de la alfabetización financiera, los estudios de Potrich et al. 2015 fueron en Brasil sobre jóvenes y el de Rai, et al., 2019 en la India. 
Tabla 3: Preguntas de las escalas Actitudes y Comportamiento financiero que ajustaron en el análisis confirmatorio

\begin{tabular}{|l|l|}
\hline Actitudes financieras: & Comportamiento Financiero: \\
\hline Es importante controlar los gastos cada mes & Pago mis deudas a tiempo \\
Es importante fijarse metas financieras para el futuro & Llevo un registro de mis gastos personales \\
Es importante ahorrar dinero cada mes & Tengo metas financieras en el largo plazo que están \\
Es importante mantenerse dentro de un presupuesto & dentro de mi presupuesto \\
Es importante invertir regularmente para lograr metas en el & Sigo cada semana o cada mes un presupuesto \\
largo plazo & Ahorro mensualmente \\
& Comparo precios cuando compro algo \\
\hline
\end{tabular}

Tabla 4: Indicadores de Bondad de Ajuste de Modelo de Alfabetización Financiera de jóvenes en Ecuador

\begin{tabular}{|l|c|}
\hline \multicolumn{1}{|c|}{ Indicador: } & Modelo General \\
\hline CMIN/DF & 2.47 \\
\hline CFI- Comparative Fit Index & 0.933 \\
\hline NFI-Normed Fit Index & 0.893 \\
\hline TLI-Tuker Lewis Index & 0.921 \\
\hline RMSEA-RMS Error of Approximation & 0.05 \\
\hline
\end{tabular}

\section{Niveles de Alfabetización financiera}

Se calcularon los puntajes obtenidos por los encuestados en cada dimensión por medio de los ítems que sí salieron significantes en el modelo. (Fig 1). Se destaca que el nivel de alfabetización financiera es de nivel medio en el $80 \%$ de los encuestados y alto en el $22 \%$, sólo el $0.5 \%$ tiene bajo nivel. En cuanto a conocimientos financieros, es bajo en un $62 \%$ de los encuestados, pero en actitudes es alto en $78 \%$, es decir son los opuestos, y el comportamiento financiero es alto para un $51 \%$ de los jóvenes, medio en un $46 \%$. En los estudios de Potrich et al.,2015 en Brasil, el nivel de conocimientos financieros fue bajo también, el nivel de actitud financiero fue alto y el nivel de comportamiento financiero fue medio en su mayoría, al igual que en este estudio. Para la encuesta de la OCDE realizada en el 2018, en promedio el nivel de conocimiento financiero fue de un $63 \%$ del máximo a alcanzar; el nivel de comportamiento financiero promedio fue de 5.3 puntos sobre 9 equivalente al $58 \%$ del máximo a alcanzar; y el nivel promedio de actitudes financieras fue de 3 sobre 5 equivalente a $60 \%$ (OECD, 2021).

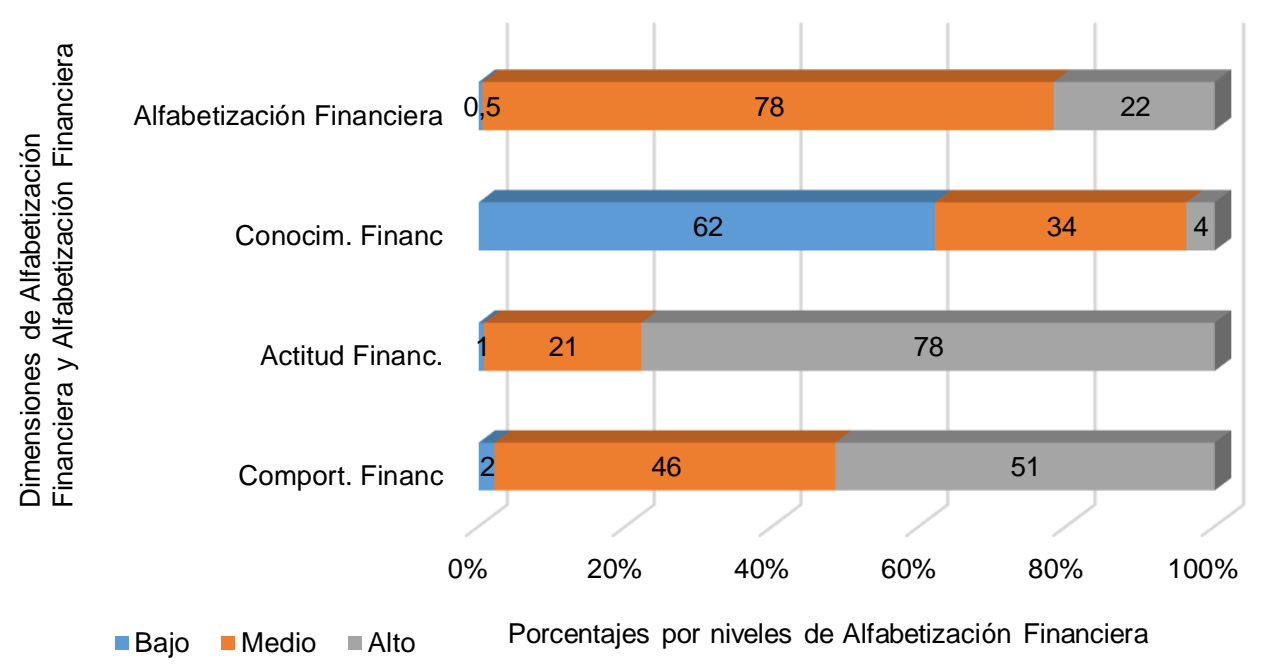

Fig. 1: Niveles de alfabetización financiera

\section{Factores sociodemográficos que influyen en la alfabetización financiera}

Para conocer los factores socioeconómicos y demográficos que podrían incidir en los niveles de la alfabetización financiera, se obtuvo un análisis discriminante por medio de un análisis ANOVA (Tabla 5). Acorde a los resultados, a diferencia de muchos estudios sobre alfabetización financiera, la variable género no tiene relación significativa con ninguna dimensión estudiada, este resultado es consistente con Despard y 
Chowa (2014) y con el estudio de la OCDE del 2018 (OECD, 2021), mientras que las variables: estado civil, actividad que realiza, si trabaja en negocio propio o en relación de dependencia, el nivel de ingresos, el nivel de estudios, tienen una relación altamente significativa con el comportamiento financiero, el conocimiento financiero y la alfabetización financiera pero no con la actitud financiera, estos factores también son consistentes con los de la OCDE, así como de Potrich et al. (2015); sobre el comportamiento financiero de los padres y si los padres les enseñan a ahorrar, tienen relación significativa con el comportamiento financiero, las actitudes financieras y la alfabetización financiera pero no con los conocimientos financieros, por lo que se debería promover más la enseñanza del ahorro en el hogar. En la tabla 5 se muestran los valores del estadístico de prueba p-value para cada variable, siendo los menores a ,005 los de relación significativamente estadística.

Tabla 5: Significancia de factores que tienen diferencias de medias con niveles de alfabetización financiera, ANOVA test.

\begin{tabular}{|l|c|c|c|c|c|c|c|c|}
\hline & $A$ & $b$ & $C$ & $D$ & $e$ & $f$ & $G$ & $H$ \\
\hline Comp. Finan. & 0.39 & 0.00 & 0.00 & 0.00 & 0.00 & 0.00 & 0.00 & 0.00 \\
\hline Act.Finan. & 0.37 & 0.62 & 0.28 & 0.66 & 0.68 & 0.22 & 0.00 & 0.00 \\
\hline Conoc. Finan. & 0.16 & 0.00 & 0.00 & 0.00 & 0.00 & 0.00 & 0.35 & 0.19 \\
\hline Alfab. Fin & 0.72 & 0.00 & 0.00 & 0.00 & 0.00 & 0.00 & 0.00 & 0.00 \\
\hline
\end{tabular}

Se ha nombrado a las columnas de la tabla 5 así: a. Género; b. Estado Civil; c. Actividad que realiza; d. Negocio Propio o Trabaja; e. Nivel de Ingresos; f. Nivel de Estudios; g. Comport. Financ. Padres; h. Padres enseñan a ahorrar. Para complementar el análisis, se aprecia que los que obtuvieron mejores niveles de alfabetización financiera fueron los casados, los varones, los que tienen los ingresos más altos y los que han culminado la universidad, indicando que a medida que maduran y van adquiriendo más responsabilidades mejoran sus niveles de alfabetización financiera.

Debido a que se identificó que los padres tienen relación con la alfabetización financiera de sus hijos, extendimos el análisis (Tabla 6) se observa que mientras más conversan los padres con los hijos sobre el ahorro, mejores niveles de alfabetización financiera presentan (en promedio), lo mismo sucede mientras mejor niveles de comportamiento financiero tienen los padres, mejores niveles tienen los hijos, se observa que aquellos que siempre conversan con sus padres sobre la importancia del ahorro son los que obtuvieron en promedio el nivel más alto de alfabetización financiera, aunque no de conocimiento financiero, ni de actitud financiera, pero sí de comportamiento financiero, lo cual va en línea con el estudio de Angulo-Ruiz y Pergelova (2015) quienes estructuraron un modelo de capacidades financieras evaluando que el comportamiento financiero de los padres influyen sobre el comportamiento de los hijos, y que las motivaciones son importantes para impulsar mejores comportamientos financieros en los hijos.

Tabla 6: Niveles de alfabetización financiera discriminando por influencia parental

\begin{tabular}{|l|l|c|c|c|c|}
\hline \multicolumn{2}{|c|}{} & $\begin{array}{c}\text { Nivel de Actitud } \\
\text { Fin. }\end{array}$ & $\begin{array}{c}\text { Nivel de Conoc. } \\
\text { Fin }\end{array}$ & $\begin{array}{c}\text { Nivel de Comp. } \\
\text { Fin }\end{array}$ & $\begin{array}{c}\text { Nivel de Alf } \\
\text { Fin }\end{array}$ \\
\cline { 3 - 6 } & & (Media) & (Media) & (Media) & Media \\
\hline \multirow{4}{*}{$\begin{array}{l}\text { Enseñanza sobre la importancia } \\
\text { del ahorro }\end{array}$} & Nunca & 4.74 & 2.4 & 3.48 & 3.54 \\
\cline { 2 - 6 } & Casi nunca & 3.99 & 2.21 & 3.32 & 3.17 \\
\cline { 2 - 6 } & A veces & 4.35 & 1.9 & 3.53 & 3.26 \\
\cline { 2 - 6 } & $\begin{array}{l}\text { Casi } \\
\text { siempre }\end{array}$ & 4.38 & 2.35 & 3.98 & 3.57 \\
\cline { 2 - 6 } & Siempre & 4.65 & 2.1 & 4.19 & 3.65 \\
\hline \multirow{3}{*}{\begin{tabular}{l} 
Tipo Nivel Comp Fin Padres \\
\cline { 2 - 6 }
\end{tabular}} & Alto & 4.6 & 2.23 & 4.31 & 3.71 \\
\cline { 2 - 6 } & Bajo & 4.18 & 2.29 & 2.94 & 3.14 \\
\cline { 2 - 6 } & Medio & 4.43 & 2.08 & 3.79 & 3.43 \\
\hline
\end{tabular}

\section{DISCUSION FINAL}

Los resultados de este estudio muestran que la alfabetización financiera no es cuestión de sólo conocimientos financieros, ni es lo mismo que educación financiera, sino que es un constructo multidimensional que se explica y puede medirse a través del comportamiento financiero, las actitudes financieras, y los conocimientos financieros de aspectos relacionados con el ahorro, la inversión, la planificación en el largo plazo, control de presupuesto y endeudamiento, así como del uso adecuado del interés simple, interés compuesto e inflación 
con respecto a las deudas e inversiones considerando el valor de las mismas en el tiempo así como su dimensión de riesgo-rendimiento y la diversificación de sus riesgos para la toma adecuada de decisiones. Esta conceptualización es consistente con otros estudios similares (Despard y Chowa, 2014; Potrich et al., 2015; Dewi et al., 2020) por ello basándose en la misma, se ha obtenido un cuestionario fiable de 16 preguntas que inicialmente eran 19; en el estudio de Potrich et al. (2015) iniciaron con 29 preguntas y en el modelo final quedaron sólo 19; en un estudio similar como el de Despard y Chowa (2014) quedaron 12 preguntas de 18 iniciales. Se aporta a la literatura con una conceptualización e instrumento de medición de alfabetización más homogéneo para un contexto de país emergente, en especial para Latinoamérica, donde los estudios han sido escasos en esta línea específica.

Los resultados de las encuestas se han clasificado por niveles de alfabetización financiera (bajo, medio y alto), obteniéndose que en su mayoría los jóvenes tienen un nivel medio de alfabetización financiera. Aunque el nivel de conocimiento financiero es bajo como en los resultados de otros estudios (Potrich et al., 2015; DiezMartínez, 2016; Moreno et al., 2017), el nivel de comportamiento financiero es medio y el de actitudes financieras es alto.

Los factores que marcan diferencias estadísticamente significativas y por tanto presentan altos niveles de alfabetización financiera y sus dimensiones son el nivel de estudios, nivel de ingresos, el estado civil, actividad a la que se dedica (estudiar o trabajar), si tiene negocio propio o trabaja en relación de dependencia, el comportamiento financiero de los padres, así como la socialización de temas de finanzas que realizan los padres o familiar más cercano a sus hijos, lo cual va en línea con Angulo-Ruiz y Pergelova (2015), sin embargo, no se evidenció diferencias por género como sí se encontró en Brasil (Potrich et al., 2015) o en Chile (Denegri Coria et al., 2016). Estos resultados indican que a medida que se va obteniendo una profesión, mejoran los ingresos, y se va adquiriendo compromisos, los niveles de alfabetización financiera también mejoran, sin embargo, se debería apuntar a que toda persona que afrontará decisiones financieras, tenga niveles adecuados de alfabetización financiera y no ser un privilegio de los que más ganan o de profesionales.

Dados estos resultados, es importante que se orienten políticas orientadas a elevar los niveles de alfabetización financiera del Ecuador, realizando mediciones periódicas con instrumentos de medición fiables como el desarrollado en este trabajo, teniendo como pilares fundamentales a los padres como agentes socializadores que más pueden incidir en los jóvenes o docentes en los centros educativos, así como se recomienda a tomar en mayor consideración aquellos grupos vulnerables como los de tercera edad, los indígenas, aquellos que viven en áreas remotas y los de menores niveles de ingresos y educación.

\section{CONCLUSIONES}

De acuerdo al trabajo presentado y a los resultados obtenidos, se pueden plantear las siguientes conclusiones principales:

1. La alfabetización financiera es un constructo multidimensional que se explica por conocimientos financieros, actitudes financieras y comportamientos financieros, de aspectos relevantes como el ahorro, la inversión, el endeudamiento, la planificación financiera y control presupuestario.

2. Se ha determinado un instrumento de medición de alfabetización financiera fiable con solo 16 preguntas que tributan al concepto de alfabetización financiera obtenido en este estudio.

3. Se ha medido los niveles de alfabetización financiera de los jóvenes encuestados, dando como resultado que la mayoría tienen un nivel medio de alfabetización financiera, derivado de un nivel bajo de conocimientos financiero, nivel alto de actitudes financieras y nivel medio de comportamiento financiero.

4. Los mejores niveles de alfabetización financiera lo tienen aquellos jóvenes con mejores ingresos, negocio propio, mejores niveles educativos, y cuyos padres muestran buenos comportamientos financieros que además socializan con sus hijos los temas financieros en el hogar.

\section{REFERENCIAS}

Angulo-Ruiz, F., y Pergelova, A., An Empowerment Model of Youth Financial Behavior, https://doi.org/10.1111/joca.12086, J Consum Aff, 49, 550-575 (2015)

Byrne, B., Structural Equation Modeling with AMOS: basic concepts, applications, and programming, 2nd. Ed., Routledge, NY, United States (2010)

Denegri, M., Araneda, K., y otros cuatro autores, Alfabetización económica y actitudes hacia la compra en universitarios posterior a un programa de educación económica, https://doi.org/10.21703/rexe.20162965814, Revista de Estudios y Experiencias en Educación, 15(29), 65-81 (2016) 
Despard, M., y Chowa, G., Testing a Measurement Model of Financial Capability among youth in Ghana, http://www.jstor.org/, The Journal of Consumer Affairs, 48(2), 301-322 (2014)

Dewi, V. I., Febrian, E., y otros tres autores, Financial literacy and its variables: The evidence from Indonesia, https://doi.org/10.14254/2071-789X.2020/13-3/9, Economics and Sociology, 13(3), 133-154 (2020)

Diez-Martínez, E., Alfabetización socioeconómica y financiera de adolescentes mexicanos del siglo XXI, Revista electrónica de investigación educativa, ISSN: 1607 4041, 18(2), 131-143 (2016)

Escobedo, M., y Hernández, J., Modelos de ecuaciones estructurales: Características, fases, construcción, aplicación y resultados, https://doi.org/10.4067/S0718-24492016000100004, Ciencia y Trabajo, 55(18), 16-22 (2016)

French, D., y McKillop, D., Financial Literacy and Over-Indebtedness in Low-Income Households, https://doi.org/10.1016/j.irfa.2016.08.004, International Review of Financial Analysis, 48, 1-11 (2016)

Garay, G., Índice de alfabetismo financiero, la cultura y la educación financiera, Perspectivas, 19(37), 23-40 (2016)

Hair, J., Black, W., y otros tres autores, Multivariate Data Analyses, seventh ed., Pearson, New Jersey, United States (2009)

Hanson, T., y Olson, P., Financial literacy and family communication patterns, https://doi.org/10.1016/j.jbef.2018.05.001, Journal of Behavioral and Experimental Finance, 19, 64-71 (2018)

Hernández, C., Cárdenas, C., Romero, P., y Hernández, C., https://doi.org/10.4067/S0718-07642017000300013, Los Padres de Familia y el Logro Académico de los Adolescentes de una Secundaria en Milpa Alta, Ciudad de México, Información Tecnológica, 28(3), 119-128 (2017)

Huston, S., Measuring financial literacy, J. Consumer Affairs, 44(2), 296-316 (2010)

Kamakia, M., Mwangi, C., y Mwangi, M., Financial Literacy and Financial Wellbeing of Public Sector Employees: A Critical Literature Review, https://doi.org/10.19044/esj.2017.v13n16p233, European Scientific Journal, ESJ, 13(16), 233 (2017)

Lusardi, A., y Mitchell, O., Planning and Financial Literacy: How Do Women Fare?, American Economic Review, 98(2), 413-417 (2008)

Lusardi, A., y Mitchell, O., Financial Literacy and Planning: Implications for Retirement Well-being, Olivia S. Mitchell y Annamaria Lusardi (eds.), Financial Literacy: Implications for Retirement Security and the Financial Marketplace, Oxford University Press, Oxford y Nueva York, 17-39 (2011a)

Lusardi, A., Financial Literacy Skills for the 21st Century: Evidence from PISA, The Journal of Consumer Affairs, Fall 2015, 639-659 (2015)

Mejía, D., Pallotta, A., Egúsquiza, E., y Palán, C., Encuesta de medición de capacidades financieras en los países andinos. Informe para Ecuador 2014 CAF, http://scioteca.caf.com/handle/123456789/74 (2015)

Moreno-García, E., García-Santillán, A., y Gutiérrez-Delgado, L., Nivel de educación financiera en escenarios de educación superior. Un estudio empírico con estudiantes del área económico-administrativa, Revista lberoamericana de Educación Superior, ISSN: 2007 2872, 8(22), 163-183 (2017)

Mungaray, A., Gonzalez, N., y Osorio, G., Educación financiera y su efecto en el ingreso en México, https://doi.org/10.22201/iiec.20078951e.2021.205.69709, Problemas Del Desarrollo, Revista Latinoamericana De Economía, 52(205), 55-78 (2021)

OECD, Improving Financial Education Efficiency, OECD-Bank of Italy Symposium on Financial Literacy, https://doi.org/10.1787/9789264108219-en, OECD Publishing, Paris (2011)

OECD, Recommendation of the Council on Financial Literacy, https://legalinstruments.oecd.org/en/instruments/OECDLEGAL-0461?_ga=2.195210476.1901847281.1621036520-1324007506.1621036519 (2021)

Potrich, A., Vieira, K., Coronel, D., y Filho, R., Financial literacy in Southern Brazil: Modeling and invariance between genders, https://doi.org/10.1016/j.jbef.2015.03.002, Journal of Behavioral Experimental Finance 6,1-12 (2015)

Potrich, A., Vieira, K., y Mendes-Da-Silva, W., Development of a financial literacy model for university students, https://doi.org/10.1108/MRR-06-2014-0143, Management Research Review, 39(3),356-376 (2016)

Rai, K., Dua, S., y Yadav, M., Association of Financial Attitude, Financial Behavior and Financial Knowledge Towards Financial Literacy: A Structural Equation Modeling Approach, https://doi.org/10.1177/2319714519826651, FIIB Business Review, 8(1), 1-10 (2019)

Raut, R., Past behavior, financial literacy and investment decision-making process of individual investors, https://doi.org/10.1108/IJOEM-07-2018-0379, International Journal of Emerging Markets,15(6), 1243-1263 (2020)

Widjaja, I., Arifin, A., y Setini, M., The Effects of Financial Literacy and subjective norms on Saving Behavior, https://doi.org/10.5267/j.msl.2020.6.030, Management Science Letters, 10, 3635-3642 (2020) 
